Homology, Homotopy and Applications, vol.5(1), 2003, pp.325-344

\title{
THE BLOCH INVARIANT AS A CHARACTERISTIC CLASS IN $B\left(S L_{2}(\mathbb{C}), \mathfrak{T}\right)$
}

\author{
JOSÉ LUIS CISNEROS-MOLINA AND JOHN D. S. JONES
}

(communicated by Jonathan Rosenberg)

\begin{abstract}
Given an orientable complete hyperbolic 3-manifold of finite volume $M$ we construct a canonical class $\alpha(M)$ in $H_{3}\left(B\left(S L_{2}(\mathbb{C}), \mathfrak{T}\right)\right)$ with $B\left(S L_{2}(\mathbb{C}), \mathfrak{T}\right)$ the $S L_{2}(\mathbb{C})$-orbit space of the classifying space for a certain family of isotropy subgroups. We prove that $\alpha(M)$ coincides with the Bloch invariant $\beta(M)$ of $M$ defined by Neumann and Yang in [13], giving with this a simpler proof that the Bloch invariant is independent of an ideal triangulation of $M$. We also give a new proof of the fact that the Bloch invariant lies in the Bloch group $B(\mathbb{C})$.
\end{abstract}

\section{Introduction}

Given a compact oriented hyperbolic 3-manifold $M$ endowed with a spin structure there is a representation $\rho: \pi_{1}(M) \rightarrow S L_{2}(\mathbb{C})$ given by the spin structure. Such representation corresponds to a map $B \rho: M \rightarrow B S L_{2}(\mathbb{C})$ where $B S L_{2}(\mathbb{C})$ is the classifying space of $S L_{2}(\mathbb{C})$ considered as a discrete group. There is a well-known invariant $\langle M\rangle$ of $M$ in $H_{3}\left(B S L_{2}(\mathbb{C}) ; \mathbb{Z}\right)$ given by the image of the fundamental class of $M$ under the homomorphism induced in homology by $B \rho$. We extend this invariant to an invariant $\alpha(M)$ when $M$ is a complete oriented hyperbolic 3-manifold of finite volume (i.e. $M$ is compact or with cusps) but in this case $\alpha(M)$ takes values in $H_{3}\left(B\left(S L_{2}(\mathbb{C}), \mathfrak{T}\right) ; \mathbb{Z}\right)$ where $B\left(S L_{2}(\mathbb{C}), \mathfrak{T}\right)$ is the $S L_{2}(\mathbb{C})$-orbit space of $E\left(S L_{2}(\mathbb{C}), \mathfrak{T}\right)$, the classifying space for $S L_{2}(\mathbb{C})$-actions with isotropy subgroups in certain subgroup-closed $S L_{2}(\mathbb{C})$-family $\mathfrak{T}$. We show that there is a canonical map $\psi: M_{+} \rightarrow B\left(S L_{2}(\mathbb{C}), \mathfrak{T}\right)$ where $M_{+}$is the one-point compactification of $M$. The invariant $\alpha(M)$ is defined as the image of the fundamental class of $M_{+}$under the homomorphism $\psi_{*}: H_{3}\left(M_{+} ; \mathbb{Z}\right) \rightarrow H_{3}\left(B\left(S L_{2}(\mathbb{C}), \mathfrak{T}\right) ; \mathbb{Z}\right)$. The invariant $\alpha(M)$ extends $\langle M\rangle$ in the following sense: There is a canonical map $k: B S L_{2}(\mathbb{C}) \rightarrow B\left(S L_{2}(\mathbb{C}), \mathfrak{T}\right)$ and when $M$ is compact $M_{+}=M$ and $\psi \simeq k \circ B \rho$. Therefore the homomorphism in homology induced by $k$ sends $\langle M\rangle$ to $\alpha(M)$.

The first author was partially supported by proyecto CONACYT G36357-E.

Received August 23, 2003, revised September 9, 2003; published on September 23, 2003.

2000 Mathematics Subject Classification: 57M27, 53R40, 19E99, 19D99

Key words and phrases: Hyperbolic 3-manifolds, Classifying space for families of isotropy subgroups, Bloch group, Bloch invariant, acyclic maps

(C) 2003, José Luis Cisneros-Molina and John D. S. Jones. Permission to copy for private use granted. 
By $[\mathbf{1 7}$, Lemma 2.2$]$ we have that $H_{3}\left(B\left(S L_{2}(\mathbb{C}), \mathfrak{T}\right) ; \mathbb{Z}\right) \cong \mathcal{P}(\mathbb{C})$ where $\mathcal{P}(\mathbb{C})$ is the pre-Bloch group. Using (degree one) ideal triangulations of $M$ Neumann and Yang defined in $[\mathbf{1 3}]$ an element $\beta(M) \in \mathcal{P}(\mathbb{C})$ called the Bloch invariant of $M$. They proved that $\beta(M)$ is independent of the ideal triangulation, showing that the generator of certain relative homology group isomorphic to $\mathbb{Z}$ is mapped to $\beta(M)$. We prove that $\alpha(M)$ coincides with the Bloch invariant $\beta(M)$. This gives a simpler proof that the Bloch invariant is independent of the ideal triangulation.

A priori, we have that $\alpha(M)$ lies in $\mathcal{P}(\mathbb{C})$ but in $[\mathbf{1 3}]$ Neumann and Yang proved that $\beta(M)$ actually lies in certain subgroup $B(\mathbb{C})$ of $\mathcal{P}(\mathbb{C})$ called the Bloch group. We also give a new proof of this fact. We do this by constructing a map $R: M_{+} \rightarrow B S L_{2}(\mathbb{C})^{+}$such that the canonical map $\psi: M_{+} \rightarrow B\left(S L_{2}(\mathbb{C}), \mathfrak{T}\right)$ factors through $B S L_{2}(\mathbb{C})^{+}$as $\psi \simeq h \circ R$, where $h: B S L_{2}(\mathbb{C})^{+} \rightarrow B\left(S L_{2}(\mathbb{C}), \mathfrak{T}\right)$ is the map induced by the canonical map $k: B S L_{2}(\mathbb{C}) \rightarrow B\left(S L_{2}(\mathbb{C}), \mathfrak{T}\right)$ by the plusconstruction. Taking the homomorphisms induced in homology we have that $\psi_{*}$ factors through $H_{3}\left(S L_{2}(\mathbb{C}) ; \mathbb{Z}\right)$ and by the Bloch-Wigner exact sequence [8, Appendix A] (see Section 7) we have that the image of $h_{*}$ is precisely the Bloch group $B(\mathbb{C})$.

The paper is organised as follows. In Section 2 we recall a well-known theorem which gives the global geometry of the aforementioned hyperbolic 3-manifolds. In Section 3 we define the classifying spaces for families of isotropy subgroups and we list some of their main properties. In Section 4 we define the invariant $\alpha(M)$. In Section 5 we recall the definitions of the Bloch group $B(\mathbb{C})$ and the Bloch invariant and in Section 6 we prove that this invariant coincides with $\alpha(M)$. In Section 7 we describe the Bloch-Wigner exact sequence and finally, in Section 8 we construct the map $R: M_{+} \rightarrow B S L_{2}(\mathbb{C})^{+}$and prove that $\alpha(M)$ lies in the Bloch group $B(\mathbb{C})$.

\section{Hyperbolic 3-manifolds}

A complete oriented hyperbolic 3-manifold $M$ is the quotient of the hyperbolic 3space $\mathcal{H}^{3}$ by a discrete, torsion-free subgroup $\Gamma$ of orientation preserving isometries.

Identify the upper half space model $\mathcal{H}^{3}$ with the set of quaternions $\{z+t \mathrm{j} \mid$ $z \in \mathbb{C}, \quad t>0\}$. The space $\mathcal{H}^{3}$ is bounded by the extended complex plane $\hat{\mathbb{C}}=$ $\mathbb{C} \cup\{\infty\}$ (Riemann sphere). The group of orientation preserving isometries of $\mathcal{H}^{3}$ is isomorphic to $P S L_{2}(\mathbb{C})=S L_{2}(\mathbb{C}) /\{ \pm I\}$ and the action of $\left(\begin{array}{ll}a & b \\ c & d\end{array}\right) \in P S L_{2}(\mathbb{C})$ in $\mathcal{H}^{3}$ is given by the linear fractional transformation

$$
\phi(w)=(a w+b)(c w+d)^{-1}, \quad w=z+t j, \quad a d-b c=1,
$$

which is the extension to $\mathcal{H}^{3}$ of the complex linear fractional transformation on $\hat{\mathbb{C}}$ given by $\left(\begin{array}{ll}a & b \\ c & d\end{array}\right)$. This action is transitive and the isotropy group of the point $(0,1) \in$ $\mathcal{H}^{3}$ is $P S U(2) \cong S O(3)$, its maximal compact subgroup. Thus the correspondence $g \mapsto g(0,1)$ for $g \in P S L_{2}(\mathbb{C})$ induces a diffeomorphism

$$
P S L_{2}(\mathbb{C}) / S O(3) \cong S L_{2}(\mathbb{C}) / S U(2) \stackrel{\cong}{\rightrightarrows} \mathcal{H}^{3} .
$$

Any discrete, torsion-free subgroup $\Gamma$ of $P S L_{2}(\mathbb{C})$ acts freely and properly dis- 
continuously on $\mathcal{H}^{3}$. Then, the quotient map

$$
\pi: \mathcal{H}^{3} \rightarrow \Gamma \backslash \mathcal{H}^{3}=M,
$$

is the universal cover and therefore $\pi_{1}(M)=\Gamma$. Note that $M$ is a $\mathbf{K}(\Gamma, 1)$, i.e. $M=B \Gamma$, the classifying space of $\Gamma$.

Two hyperbolic 3-manifolds $M=\Gamma \backslash \mathcal{H}^{3}$ and $M^{\prime}=\Gamma^{\prime} \backslash \mathcal{H}^{3}$ are isometric by an orientation-preserving isometry if and only if $\Gamma$ and $\Gamma^{\prime}$ are conjugate in $P S L_{2}(\mathbb{C})$ [14, Thm. 8.1.5]. To such an hyperbolic 3-manifold we can associate a representation $\rho: \pi_{1}(M)=\Gamma \rightarrow P S L_{2}(\mathbb{C})$ which is canonical up to equivalence.

Using the diffeomorphism (1) we have that any complete, oriented, hyperbolic 3 -manifold $M$ is given by the double coset space

$$
M=\Gamma \backslash P S L_{2}(\mathbb{C}) / S O(3),
$$

with $\Gamma=\pi_{1}(M)$ a discrete torsion-free subgroup. The canonical representation $\Gamma \rightarrow P S L_{2}(\mathbb{C})$ is given by the inclusion and it can be lifted to a representation $\rho: \Gamma \rightarrow S L_{2}(\mathbb{C})[6$, Prop. 3.1.1]. There is a one-to-one correspondence between such lifts and spin structures on $M$. Hence, any complete, oriented hyperbolic 3-manifold $M$ equipped with a spin structure can be seen as the double coset space

$$
M=\Gamma \backslash S L_{2}(\mathbb{C}) / S U(2),
$$

where we identify $\Gamma$ with a subgroup of $S L_{2}(\mathbb{C})$ using the representation $\rho: \Gamma \rightarrow$ $S L_{2}(\mathbb{C})$ which corresponds to the spin structure.

Throughout this paper, $M$ will denote a non-compact orientable complete hyperbolic 3-manifold of finite volume. The global geometry of such manifolds is given in the following theorem, see for instance [14, p. 647 Cor. 4 and Thm. 10.2.1].

Theorem 2.1. Let $M$ be an orientable complete hyperbolic 3-manifold of finite volume. Then there is a compact 3-manifold-with-boundary $M_{0}$ in $M$ such that $M-M_{0}$ is the disjoint union of a finite number of cusps. Each cusp of $M$ is diffeomorphic to $T^{2} \times(0, \infty)$, where $T^{2}$ denotes the 2-torus.

Remark 2.2. Let $M$ be an oriented complete hyperbolic 3-manifold of finite volume with $d$ cusps. Take a base point $x_{0}$ in $M$ and a point $x_{i}$ in a torus section $T_{i}^{2}$ of the end corresponding to the $i$-th cusp $(i=1, \ldots, d)$. Let $q_{i}(t)(0 \leqslant t \leqslant 1)$ be a path in $M$ with $q_{i}(0)=x_{0}$ and $q_{i}(1)=x_{i}$. Consider the homomorphism $\left(q_{i}\right)_{\#}: \pi_{1}\left(T_{i}^{2}, x_{i}\right) \rightarrow \pi_{1}\left(M, x_{0}\right)$ induced by $q_{i}$ and put $\Gamma_{i}=\left(q_{i}\right)_{\#}\left(\pi_{1}\left(T_{i}^{2}, x_{i}\right)\right)$. The subgroups $\Gamma_{i}$ are called the peripheral torus subgroups of $\Gamma$. The image of $\Gamma_{i}$ under the representation $\rho: \Gamma \rightarrow S L_{2}(\mathbb{C})$ given by the inclusion is a free abelian group of rank 2 of $S L_{2}(\mathbb{C})$. The subgroups $\Gamma_{i}$ consist only of parabolic elements, that is, they fix no points of $\mathcal{H}^{3}$ and fix a unique point of the boundary $\hat{\mathbb{C}}$ of $\mathcal{H}^{3}$. All the elements in $\Gamma_{i}$ have as a fixed point the corresponding cusp point $[\mathbf{1 8}, \S 4.5]$. Since the action of $S L_{2}(\mathbb{C})$ in $\hat{\mathbb{C}}$ is transitive and the conjugates of parabolic isometries are parabolic $[\mathbf{1 4}$, p. 141] we can assume that the fixed point is the point at infinity $\infty$ which we denote by its homogeneous coordinates $\infty=[1,0]$ and therefore the subgroups $\Gamma_{i}$ are conjugate to a group of matrices of the form $\left(\begin{array}{cc} \pm 1 & \lambda \\ 0 & \pm 1\end{array}\right)$, with $\lambda \in \mathbb{C}$. 


\section{Classifying spaces for families of isotropy subgroups}

Let $G$ be a group and let $\mathfrak{s u b}(G)$ denote the set of all subgroups of $G$. A subset $\mathfrak{F}$ of $\mathfrak{s u b}(G)$ is a subgroup-closed $G$-family if each subgroup of each element of $\mathfrak{F}$ belongs to $\mathfrak{F}$ and, moreover, $\mathfrak{F}$ is closed under taking conjugates by elements of $G$. Let $\left\{H_{i}\right\}_{i \in I}$ be subgroups of $G$, we denote by $\mathfrak{F}\left(H_{i}\right)$ the subgroup-closed $G$-family consisting of all the subgroups of the $\left\{H_{i}\right\}_{i \in I}$ and all their conjugates by elements of $G$.

Let $X$ be a $G$-space. For each subgroup $H$ of $G$, we define the set $X^{H}$ consisting of points of $X$ fixed by all of $H$.

A classifying space for $G$-actions with isotropy subgroups in $\mathfrak{F}$, or a space of type $E(G, \mathfrak{F})$, is a $G$-space with the following properties: for each $H \in \mathfrak{F}$, $X^{H}$ is contractible and for each $H \in \mathfrak{s u b}(G)-\mathfrak{F}, X^{H}$ is empty.

The $G$-space $E(G, \mathfrak{F})$ has all its isotropy subgroups in $\mathfrak{F}$ and it has the following universal property. Given any $G$-space $X$ with isotropy subgroups in $\mathfrak{F}$ there exist a $G$-map

$$
X \rightarrow E(G, \mathfrak{F})
$$

which is unique up to $G$-homotopy $[\mathbf{1 9}$, Rmk. 7.2.1], [20, Thm. 6.6]. The classifying space $E(G, \mathfrak{F})$ is unique up to $G$-homotopy equivalence [7, Prop. 2.2], [19, Rmk. 7.2.2].

There are different ways of constructing the spaces $E(G, \mathfrak{F})$. We consider the following two:

1. There exist a countable system $\left\{H_{i}\right\}_{i \in I}$ of subgroups $H_{i} \in \mathfrak{F}$ such that every group in $\mathfrak{F}$ is conjugate to an $H_{i}$, that is, $\mathfrak{F}=\mathfrak{F}\left(H_{i}\right)$. Let $E_{i}=G / H_{i} * G / H_{i} * \ldots$ be the join of a countable number of copies of $G / H_{i}$. Then $E(G, \mathfrak{F})=*_{i \in I} E_{i}$ is the join of the $E_{i}, i \in I$, with the Milnor topology [19, Prop. 7.2.1].

2. Let $\Delta$ be any $G$-set such that $\mathfrak{F}$ is precisely the set of subgroups of $G$ which fix at least one point of $\Delta$. Then $E(G, \mathfrak{F})=\Delta * \Delta * \ldots$, the join of a countably number of copies of $\Delta$ [7, Prop. 2.2].

Example 3.1. Consider $S L_{2}(\mathbb{C})$ as a discrete group. Since the action of $S L_{2}(\mathbb{C})$ on the universal bundle $E S L_{2}(\mathbb{C})$ is free and $E S L_{2}(\mathbb{C})$ is contractible we have that $E S L_{2}(\mathbb{C})$ is a space of type $E\left(S L_{2}(\mathbb{C}),\{1\}\right)$.

Example 3.2. Consider the sphere $S^{2}$ as the extended complex numbers $\hat{\mathbb{C}}$. We have that $S L_{2}(\mathbb{C})$ acts triply transitively on $S^{2}$. Let $T$ be the isotropy subgroup of $\infty, T$ consists of the matrices of the form $\left(\begin{array}{cc}z & a \\ 0 & z^{-1}\end{array}\right)$, with $a, z \in \mathbb{C}$ and $z \neq 0$. Hence we have a diffeomorphism given by

$$
\begin{aligned}
S L_{2}(\mathbb{C}) / T & \cong \\
g T & \mapsto g \cdot \infty .
\end{aligned}
$$

Let $J\left(S^{2}\right)=S^{2} * S^{2} * \ldots$, the join of a countable number of copies of $S^{2}$. By construction 1 of $E(G, \mathfrak{F})$ we have that $J\left(S^{2}\right)$ is an $E\left(S L_{2}(\mathbb{C}), \mathfrak{F}(T)\right.$ ). 
Example 3.3. Let $\Gamma$ be a discrete torsion-free subgroup of $S L_{2}(\mathbb{C})$. The action of $\Gamma$ on the hyperbolic 3 -space $\mathcal{H}^{3}$ is free and since $\mathcal{H}^{3}$ is contractible, it is a space of type $E(\Gamma,\{1\})$.

Let $M=\Gamma \backslash \mathcal{H}^{3}$ be a non-compact orientable complete hyperbolic 3-manifold of finite volume. Let $\pi: \mathcal{H}^{3} \rightarrow \Gamma \backslash \mathcal{H}^{3}=M$ be the universal cover of $M$. Let $\mathcal{C}$ be the set of cusp points, that is, the preimages of cusps of $M$ in $\partial \overline{\mathcal{H}^{3}}=\hat{\mathbb{C}}$, or, equivalently, fixed points of parabolic elements of $\Gamma$ and let $\hat{Y}=\mathcal{H}^{3} \cup \mathcal{C}$. The action of $\Gamma$ on $\hat{Y}$ is no longer free. The cusp points have as isotropy subgroups the peripheral torus subgroups $\Gamma_{1}, \ldots, \Gamma_{d}$ of $\Gamma$ or their conjugates; therefore, by the definition of $E(\Gamma, \mathfrak{F})$, we have that $\hat{Y}$ is a $E\left(\Gamma, \mathfrak{F}\left(\Gamma_{1}, \ldots, \Gamma_{d}\right)\right)$.

We list some results about $E(G, \mathfrak{F})$ :

Proposition 3.4 ([7, Cor. 2.3]). If $\mathfrak{F}_{1} \subseteq \mathfrak{F}_{2}$ are subgroup-closed $G$-families, then there exist a $G$-map $E\left(G, \mathfrak{F}_{1}\right) \rightarrow E\left(G, \mathfrak{F}_{2}\right)$ unique up to $G$-homotopy.

Corollary 3.5. $\quad$ - There is a $S L_{2}(\mathbb{C})$-map $\ell: E S L_{2}(\mathbb{C}) \rightarrow J\left(S^{2}\right)$ unique up to $S L_{2}(\mathbb{C})$-homotopy.

- There is a $\Gamma$-map $l: \mathcal{H}^{3} \rightarrow \hat{Y}$ unique up to $\Gamma$-homotopy.

Let $H$ be a subgroup of $G$. For a $G$-space $X$, let $\operatorname{res}_{H} X$ be the $H$-space obtained by restricting the group action. If $\mathfrak{F}$ is a subgroup-closed $G$-family, let $\mathfrak{F} / H=$ $\{L \cap H \mid L \in \mathfrak{F}\}$ be the induced family of subgroups of $H$.

Proposition 3.6 ([19, Prop. 7.2.4]).

$$
\operatorname{res}_{H} E(G, \mathfrak{F})=E(H, \mathfrak{F} / H) .
$$

Corollary 3.7. 1. $\operatorname{res}_{\Gamma} E S L_{2}(\mathbb{C})$ is $\Gamma$-homotopy equivalent to $\mathcal{H}^{3}$.

2. There is a $\Gamma$-map $\hat{Y} \rightarrow \operatorname{res}_{\Gamma} J\left(S^{2}\right)$ unique up to $\Gamma$-homotopy.

Proof. Claim 1 is a direct consequence of Proposition 3.6. Also by Proposition 3.6 we have that $\operatorname{res}_{\Gamma} J\left(S^{2}\right)$ is an $E(\Gamma, \mathfrak{F}(T) / \Gamma)$. By Example $3.3 \hat{Y}$ is an $\mathfrak{F}\left(\Gamma_{1}, \ldots, \Gamma_{d}\right)$ where $\Gamma_{1}, \ldots, \Gamma_{d}$ are the peripheral torus subgroups. We have that $\mathfrak{F}\left(\Gamma_{1}, \ldots, \Gamma_{d}\right) \subset \mathfrak{F}(T) / \Gamma$ and the result follows by Proposition 3.4.

Following this, we give a simplicial version of the map $\ell: E S L_{2}(\mathbb{C}) \rightarrow J\left(S^{2}\right)$ of Corollary 3.5 which we use in following sections.

Example 3.8. Consider the universal bundle $E S L_{2}(\mathbb{C})$ as the geometric realisation of the simplicial complex where an $n$-simplex is an $(n+1)$-tuple $\left(g_{0}, \ldots, g_{n}\right)$ with $g_{i} \in S L_{2}(\mathbb{C})$, and the $i$-th face (resp., degeneracy) of such a simplex is obtained by omitting (resp., repeating) $g_{i}$. The action of $g \in S L_{2}(\mathbb{C})$ gives the simplex $\left(g g_{0}, \ldots, g g_{n}\right)$. The infinite join $J\left(S^{2}\right)$ is the geometric realisation of the simplicial set whose $n$-simplices are the $n+1$-tuples $\left(a_{0}, \ldots, a_{n}\right)$ of elements of $S^{2}=\hat{\mathbb{C}}$ and the $i$-th face (resp., degeneracy) of such a simplex is obtained by omitting (resp., repeating) $a_{i}$. The action of $g \in S L_{2}(\mathbb{C})$ gives the simplex $\left(g a_{0}, \ldots, g a_{n}\right)$. Hence the 
map $\ell: E S L_{2}(\mathbb{C}) \rightarrow J\left(S^{2}\right)$ is the (geometric realisation of the) $S L_{2}(\mathbb{C})$-equivariant simplicial map

$$
\left(g_{0}, \ldots, g_{n}\right) \rightarrow\left(g_{0} \cdot \infty, \ldots, g_{n} \cdot \infty\right)
$$

Choosing a different basepoint instead of $\infty$ by Corollary 3.5 we get a $S L_{2}(\mathbb{C})$ homotopic map.

\section{The invariant $\alpha(M)$ of $M$}

To simplify notation, if $G$ is a group and $X$ is a $G$-space, we denote the space of orbits $G \backslash X$ by $X_{G}$. Also for the rest of the paper, let $\mathfrak{T}=\mathfrak{F}(T)$ with $T$ as in Example 3.2 and $B\left(S L_{2}(\mathbb{C}), \mathfrak{T}\right)=E\left(S L_{2}(\mathbb{C}), \mathfrak{T}\right)_{S L_{2}(\mathbb{C})}$.

Let $M$ be an orientable complete hyperbolic 3-manifold of finite volume, choosing a spin structure on $M$ we have that $M=\Gamma \backslash \mathcal{H}^{3}$ for some discrete torsion-free subgroup of $S L_{2}(\mathbb{C})$. Let $\hat{Y}=\mathcal{H}^{3} \cup \mathcal{C}$ with $\mathcal{C}$ the set of cusp points and let $\hat{M}=\Gamma \backslash \hat{Y}$. If $M$ is compact $\mathcal{C}=\emptyset$ and $\hat{M}=M$, if $M$ is non-compact we have that $\hat{M}$ is the end-compactification of $M$ which is the result of adding the cusps of $M$.

By Corollary 3.7-1, we have that $M=\Gamma \backslash \mathcal{H}^{3}$ is homotopically equivalent to $E S L_{2}(\mathbb{C})_{\Gamma}=B \Gamma$ and by Corollary $3.7-2$ there is a $\Gamma$-map

$$
\hat{M} \rightarrow J\left(S^{2}\right)_{\Gamma}=E\left(S L_{2}(\mathbb{C}), \mathfrak{T}\right)_{\Gamma}
$$

which is unique up to $\Gamma$-homotopy. We also have that $E S L_{2}(\mathbb{C})_{S L_{2}(\mathbb{C})}=B S L_{2}(\mathbb{C})$. Combining this with Corollary 3.5 and taking the quotients by $S L_{2}(\mathbb{C})$ and $\Gamma$ we get the following commutative diagram

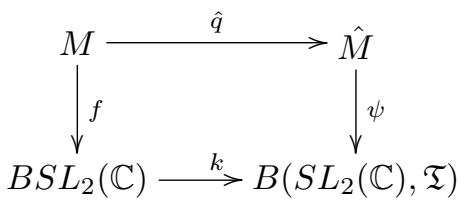

where $\hat{q}$ (resp., $k$ ) is the map induced by the map $l$ (resp. $\ell$ ) of Corollary 3.5 on the sets of $\Gamma\left(\right.$ resp. $\left.S L_{2}(\mathbb{C})\right)$ orbits; $f=B \rho: B \Gamma \rightarrow B S L_{2}(\mathbb{C})$ is the map between classifying spaces which on fundamental groups induces the canonical representation $\rho: \Gamma \rightarrow S L_{2}(\mathbb{C})$ of $M$, and $\psi$ is given by the composition

$$
\psi: \hat{M} \rightarrow E\left(S L_{2}(\mathbb{C}), \mathfrak{T}\right)_{\Gamma} \rightarrow B\left(S L_{2}(\mathbb{C}), \mathfrak{T}\right) .
$$

The map $\psi$ induces an homomorphism

$$
\psi_{*}: H_{3}(\hat{M} ; \mathbb{Z}) \rightarrow H_{3}\left(B\left(S L_{2}(\mathbb{C}), \mathfrak{T}\right) ; \mathbb{Z}\right) .
$$

Let $[\hat{M}]$ denote the fundamental class of $\hat{M}$ in $H_{3}(\hat{M} ; \mathbb{Z})$. We denote by $\alpha(M)$ the canonical class in $H_{3}\left(B\left(S L_{2}(\mathbb{C}), \mathfrak{T}\right) ; \mathbb{Z}\right)$ given by

$$
\alpha(M)=\psi_{*}([\hat{M}]) .
$$




\section{The Bloch invariant}

In the present section, following [13], we shall define the Bloch invariant of a non-compact orientable complete hyperbolic 3-manifold of finite volume which has an ideal triangulation.

The pre-Bloch group $\mathcal{P}(F)$ of a field $F$ is the quotient of the free abelian group generated by the formal symbols $[x], x \in F \cup\{\infty\}$ by the following relations

$$
\begin{gathered}
{[0]=[1]=[\infty]=0} \\
{[x]-[y]+[y / x]-\left[\left(1-x^{-1}\right) /\left(1-y^{-1}\right)\right]+[(1-x) /(1-y)]=0 .}
\end{gathered}
$$

The Bloch group $B(F)$ is the kernel of the map

$$
\begin{aligned}
\mathcal{P}(F) & \stackrel{\lambda}{\rightarrow} F^{\times} \\
{[x] } & \mapsto F^{\times} \\
& 2(x \wedge(1-x)),
\end{aligned}
$$

where $F^{\times}$is the multiplicative group of $F$.

Let $\overline{\mathcal{H}}^{3}=\mathcal{H}^{3} \cup \hat{\mathbb{C}}$ be the standard compactification of the hyperbolic 3 -space $\mathcal{H}^{3}$. We shall refer to the elements of the boundary $\widehat{\mathbb{C}}$ of $\mathcal{H}^{3}$ as points at infinity.

An ideal tetrahedron (or ideal simplex) is a tetrahedron in $\overline{\mathcal{H}}^{3}$ with all its vertices in $\hat{\mathbb{C}}$. Let $\triangle\left(a_{0}, a_{1}, a_{2}, a_{3}\right)$ be an ideal tetrahedron with vertices $a_{0}, a_{1}, a_{2}, a_{3}$. The group $P S L_{2}(\mathbb{C})$ acts on $\hat{\mathbb{C}}$ by fractional linear transformations and the action is triply transitive. Therefore, there exists an element $g \in P S L_{2}(\mathbb{C})$ such that

$$
g \cdot a_{0}=0, \quad g \cdot a_{1}=\infty, \quad g \cdot a_{2}=1, \quad g \cdot a_{3}=z
$$

where $z=\left[a_{0}: a_{1}: a_{2}: a_{3}\right]$ is the cross-ratio of the vertices given by

$$
\left[a_{0}: a_{1}: a_{2}: a_{3}\right]=\frac{\left(a_{0}-a_{3}\right)\left(a_{1}-a_{2}\right)}{\left(a_{0}-a_{2}\right)\left(a_{1}-a_{3}\right)} .
$$

Therefore the congruence classes of ideal tetrahedra are parameterised by $z \in$ $\mathbb{C}-\{0,1\}$. We extend the definition of the cross-ratio to $\left[a_{0}: a_{1}: a_{2}: a_{3}\right]=0$ whenever $a_{i}=a_{j}$ for some $i \neq j$.

Let $M$ be a non-compact orientable complete hyperbolic 3-manifold of finite volume. An ideal triangulation for $M$ is a triangulation where all the tetrahedra $\triangle_{i}$ are ideal tetrahedra.

Remark 5.1. In [9] Epstein and Penner proved that these kind of manifolds can be decomposed into convex ideal polyhedra, but to the best of our knowledge it is still unknown whether this decomposition can be subdivided into an ideal triangulation (compare $[13, \S 10]$ ).

For this reason, from now on we shall assume that when $M$ is a non-compact orientable complete hyperbolic 3-manifold of finite volume it has an ideal triangulation.

Let $M$ be an hyperbolic 3-manifold and let $\triangle_{1}, \ldots, \triangle_{n}$ be the ideal tetrahedra of an ideal triangulation of $M$. Let $z_{i} \in \mathbb{C}$ be the parameter of $\triangle_{i}$ for each $i$. These parameters define an element $\beta(M)=\sum_{i=1}^{n}\left[z_{i}\right]$ in the pre-Bloch group. The element $\beta(M) \in \mathcal{P}(\mathbb{C})$ is called the Bloch invariant of $M$. 
Remark 5.2. Neumann and Yang defined the Bloch invariant using degree one ideal triangulations, in that way it is defined for all hyperbolic 3-manifolds of finite volume, even the compact ones, see $[\mathbf{1 3}, \S 2]$ for details.

In [13, Thm. 1.1] Neumann and Yang proved that $\beta(M)$ depends only on $M$ and not on the triangulation, and that it actually lies in the Bloch group $B(\mathbb{C})$. In the following section, we give a simpler proof of the independence of the Bloch invariant of the ideal triangulation proving that $\alpha(M)=\beta(M)$. In section 8 we give another proof that $\alpha(M)=\beta(M) \in B(\mathbb{C})$.

\section{The invariant $\alpha(M)$ is the Bloch invariant}

In this section we prove that the characteristic class $\alpha(M)$ of an orientable complete hyperbolic 3-manifold of finite volume $M$ equals its Bloch invariant $\beta(M)$, in particular, this gives a new proof that the Bloch invariant is independent of the ideal triangulation, or from a different point of view, we can compute $\alpha(M)$ using an ideal triangulation of $M$. $\mathfrak{T})$.

For the rest of the paper we shall consider $J\left(S^{2}\right)_{S L_{2}(\mathbb{C})}$ as the model for $B\left(S L_{2}(\mathbb{C})\right.$,

Firstly, by [17, Lemma 2.2] (but using $S L_{2}(\mathbb{C})$ instead of $G L_{2}(\mathbb{C})$ ) we have that $H_{3}\left(B\left(S L_{2}(\mathbb{C}), \mathfrak{T}\right) ; \mathbb{Z}\right)=H_{3}\left(J\left(S^{2}\right)_{S L_{2}(\mathbb{C})} ; \mathbb{Z}\right) \cong \mathcal{P}(\mathbb{C})$, hence $\alpha(M) \in \mathcal{P}(\mathbb{C})$. The isomorphism is given by

$$
\left(a_{0}, a_{1}, a_{2}, a_{3}\right)_{S L_{2}(\mathbb{C})} \mapsto\left[a_{0}: a_{1}: a_{2}: a_{3}\right],
$$

$\left(a_{0}, a_{1}, a_{2}, a_{3}\right)_{S L_{2}(\mathbb{C})}$ denotes the $S L_{2}(\mathbb{C})$-orbit of the 3 -simplex $\left(a_{0}, a_{1}, a_{2}, a_{3}\right)$ and $\left[a_{0}: a_{1}: a_{2}: a_{3}\right]$ is the cross-ratio of the $a_{i} \in \hat{\mathbb{C}}, i=0,1,2,3$.

Theorem 6.1. Let $M$ be an orientable complete hyperbolic 3-manifold of finite volume. Then $\alpha(M)=\beta(M)$.

Proof. Let $M$ be a non-compact orientable complete hyperbolic 3-manifold of finite volume. Let $\pi: \mathcal{H}^{3} \rightarrow \Gamma \backslash \mathcal{H}^{3}=M$ be the universal cover of $M$. Then $M$ lifts to an exact, convex, fundamental, ideal polyhedron $P$ for $\Gamma$ [14, Thm. 11.2.1]. An ideal triangulation of $M$ gives a decomposition of $P$ into a finite number of ideal tetrahedra. Since $\mathcal{P}=\{g P \mid g \in \Gamma\}$ is an exact tessellation of $\mathcal{H}^{3}$ [14, Thm. 6.7.1], this decomposition of $P$ gives an ideal triangulation of $\mathcal{H}^{3}$.

As in Example 3.3, let $\mathcal{C}$ be the set of cusp points and let $\hat{Y}=\mathcal{H}^{3} \cup \mathcal{C}$, which is the result of adding the vertices of the ideal tetrahedra of the ideal triangulation of $\mathcal{H}^{3}$. Hence we can consider $\hat{Y}$ as a simplicial complex with vertices given by the cusp points.

To prove the theorem, we use the simplicial version of the $\Gamma$-map $\hat{Y} \rightarrow J\left(S^{2}\right)$ given in Corollary 3.7-2. Consider $J\left(S^{2}\right)$ as the geometric realization of a simplicial complex as in Example 3.8. Considering $\hat{Y}$ as the geometric realisation of its ideal triangulation and since its vertices are the cusp points $\mathcal{C} \subset \hat{\mathbb{C}}$ we have that the $\Gamma$-map $\hat{Y} \rightarrow J\left(S^{2}\right)$ is given by the (geometric realisation of the) $\Gamma$-equivariant simplicial 
map

$$
\begin{aligned}
\hat{Y} & \rightarrow J\left(S^{2}\right) \\
\left(a_{0}, a_{1}, a_{2}, a_{3}\right) & \mapsto\left(a_{0}, a_{1}, a_{2}, a_{3}\right) .
\end{aligned}
$$

The composition (4) induces on 3 -chains the map

$$
\begin{gathered}
C_{3}(\hat{M}) \rightarrow C_{3}\left(J\left(S^{2}\right)_{S L_{2}(\mathbb{C})}\right) \\
\left(a_{0}, a_{1}, a_{2}, a_{3}\right)_{\Gamma} \mapsto\left(a_{0}, a_{1}, a_{2}, a_{3}\right)_{S L_{2}(\mathbb{C})}=(0, \infty, 1, z)_{S L_{2}(\mathbb{C})} .
\end{gathered}
$$

where $\left(a_{0}, a_{1}, a_{2}, a_{3}\right)_{\Gamma}\left(\operatorname{resp} .\left(a_{0}, a_{1}, a_{2}, a_{3}\right)_{S L_{2}(\mathbb{C})}\right)$ denotes the $\Gamma$-orbit (resp. $S L_{2}(\mathbb{C})$ orbit) of the 3 -simplex $\left(a_{0}, a_{1}, a_{2}, a_{3}\right)$ and $z=\left[a_{0}: a_{1}: a_{2}: a_{3}\right]$.

If $\triangle_{i}=\triangle\left(a_{0}^{i}, a_{1}^{i}, a_{2}^{i}, a_{3}^{i}\right), i=1, \ldots, n$, are the ideal tetrahedra of an ideal triangulation of $M$ and $z_{i}=\left[a_{0}^{i}: a_{1}^{i}: a_{2}^{i}: a_{3}^{i}\right] \in \mathbb{C}$ is the parameter of $\triangle_{i}$ for each $i$, now is clear that under the homomorphism (5) the fundamental class $[\hat{M}]$ is sent to the Bloch invariant $\beta(M)$

$$
\begin{gathered}
H_{3}(\hat{M}) \rightarrow H_{3}\left(J\left(S^{2}\right)_{\Gamma}\right) \rightarrow \mathcal{P}(\mathbb{C}) \\
{[\hat{M}] \mapsto \sum_{i=1}^{n}\left(a_{0}^{i}, a_{1}^{i}, a_{2}^{i}, a_{3}^{i}\right)_{\Gamma} \mapsto \sum_{i=1}^{n}\left(a_{0}^{i}, a_{1}^{i}, a_{2}^{i}, a_{3}^{i}\right)_{\left.S L_{2}(\mathbb{C})\right)}=\sum_{i=1}^{n}\left[z_{i}\right] .}
\end{gathered}
$$

For the case when $M$ is compact, following $[\mathbf{1 3}, \S 2]$ choose a degree one ideal triangulation of $M$ given by a geometric 3-cycle $Y$ and a map $f: Y-Y^{(0)} \rightarrow M$ with $|Y|=M$. As in [13, §4], form the pull-back covering

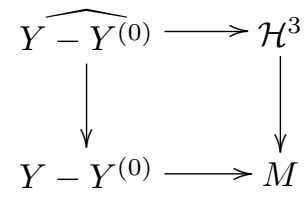

and complete to get a simplicial complex $\hat{Y}$ with a $\Gamma$-action (which is free except maybe at the vertices). By Corollary 3.7 there is a $\Gamma$-map $\hat{Y} \rightarrow J\left(S^{2}\right)$ and we can substitute $\mathcal{H}^{3}$ by $E S L_{2}(\mathbb{C})$. Taking the quotients by $S L_{2}(\mathbb{C})$ and $\Gamma$ we get the following commutative diagram

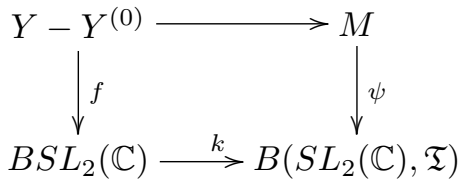

since $\Gamma \backslash \hat{Y}=Y=M$. The rest of the proof is as for the non-compact case using this $\hat{Y}$.

Remark 6.2. Recall that by Theorem $2.1 M$ is homotopically equivalent to $M_{0}$ therefore we can think that the map $\hat{q}: M_{0} \rightarrow \hat{M}$ collapses each torus component of $M_{0}$ to a point. On the other hand, consider the quotient map $c: \hat{M} \rightarrow M_{+}$from the end-compactification to the one-point-compactification of $M$ which consists in identifying all the cusps points of $\hat{M}$ to a single point. Since the action of $G$ on 
$J\left(S^{2}\right)$ identifies all the 0 -simplices, all the cusps points of $\hat{M}$ are mapped to the unique 0-simplex of $J\left(S^{2}\right)_{S L_{2}(\mathbb{C})}$ under the map $\psi: \hat{M} \rightarrow J\left(S^{2}\right)_{S L_{2}(\mathbb{C})}$, thus the map $\psi$ descends to a map $M_{+} \rightarrow J\left(S^{2}\right)_{S L_{2}(\mathbb{C})}$ which we shall also call $\psi$. Since the map $c$ induces an isomorphism in $H_{3}$ we also have that $\alpha(M)=\psi_{*}\left(\left[M_{+}\right]\right)=\beta(M)$ with $\left[M_{+}\right]$the fundamental class of $M_{+}$. Hence, diagram (3) is equivalent to the following with $q=c \circ \hat{q}$

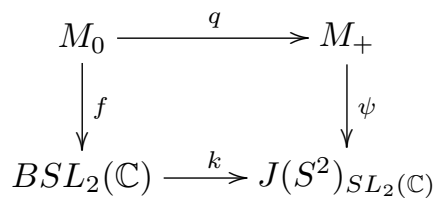

\section{The Bloch-Wigner exact sequence}

In the present section we describe the Bloch-Wigner exact sequence $[\mathbf{8}$, Appendix A] which will help us in the next section to prove that $\alpha(M)=\beta(M) \in B(\mathbb{C})$.

Recall that for a (discrete) group $G$ its (Eilenberg-Mac Lane) cohomology groups $H_{E M}^{n}(G, A)$ with coefficients in a $G$-module $A$ is the homology of the complex

$$
C^{n}(G, A)=\left\{f: G^{n+1} \rightarrow A \mid g f\left(g_{0}, \ldots, g_{n}\right)=f\left(g g_{0}, \ldots, g g_{n}\right)\right\}
$$

with coboundary $\delta: C^{n}(G, A) \rightarrow C^{n+1}(G, A)$ given by

$$
\delta(f)\left(g_{0}, \ldots, g_{n+1}\right)=\sum_{i=0}^{n+1}(-1)^{i} f\left(g_{0}, \ldots, \hat{g}_{i}, \ldots, g_{n+1}\right),
$$

where $\hat{g}_{i}$ indicates that $g_{i}$ is omitted.

Lemma 7.1. Let $G=S L_{2}(\mathbb{C})$. Consider the function given by

$$
\begin{gathered}
\bar{\tau}: G \times G \times G \times G \rightarrow \mathcal{P}(\mathbb{C}) \\
\bar{\tau}\left(g_{0}, g_{1}, g_{2}, g_{3}\right)=\left[\left[g_{0} \cdot \infty: g_{1} \cdot \infty: g_{2} \cdot \infty: g_{3} \cdot \infty\right]\right], \quad \infty \in \hat{\mathbb{C}},
\end{gathered}
$$

where $\left[a_{0}: a_{1}: a_{2}: a_{3}\right]$ with $a_{i} \in \hat{\mathbb{C}}$ is the cross-ratio given by (7) and a cross-ratio of non-distinct points is taken as zero. Then $\bar{\tau}$ represents a cohomology class in $H_{E M}^{3}\left(S L_{2}(\mathbb{C}), \mathcal{P}(\mathbb{C})\right)$ which we denote by $\tau$.

Proof. Clearly $\bar{\tau}$ is invariant under the left diagonal action of $G$. First we are going to show that it is a cocycle of the complex $C^{\bullet}(G, \mathcal{P}(\mathbb{C}))$. Since the action of $S L_{2}(\mathbb{C})$ in $\hat{\mathbb{C}}$ is triply transitive we can assume that

$$
\left(g_{0} \cdot \infty, g_{1} \cdot \infty, g_{2} \cdot \infty, g_{3} \cdot \infty, g_{4} \cdot \infty\right)=(0, \infty, 1, x, y) .
$$

Thus, we have

$$
\begin{aligned}
\delta(\bar{\tau})\left(g_{0}, g_{1}, g_{2}, g_{3}, g_{4}\right)=[\infty: 1: x: y]-[0: 1: x: y]+[0: \infty: x: y] & \\
& -[0: \infty: 1: y]+[0: \infty: 1: x],
\end{aligned}
$$


and applying the definition of the cross-ratio we get

$$
\delta(\bar{\tau})\left(g_{0}, g_{1}, g_{2}, g_{3}, g_{4}\right)=\left[\frac{1-x}{1-y}\right]-\left[\frac{1-x^{-1}}{1-y^{-1}}\right]+\left[\frac{y}{x}\right]-[y]+[x],
$$

which is the 5 -term relation which defines $\mathcal{P}(\mathbb{C})$ and therefore it is zero. Hence, $\tau$ represents a class in $H_{E M}^{3}\left(S L_{2}(\mathbb{C}), \mathcal{P}(\mathbb{C})\right)$.

Evaluating the class $\tau$ of Lemma 7.1 on homology we get a homomorphism

$$
\begin{gathered}
H_{3}^{E M}\left(S L_{2}(\mathbb{C}), \mathbb{Z}\right) \stackrel{\tau}{\rightarrow} \mathcal{P}(\mathbb{C}) \\
\left(g_{0}, g_{1}, g_{2}, g_{3}\right)_{S L_{2}(\mathbb{C})} \mapsto\left[\left[g_{0} \cdot \infty: g_{1} \cdot \infty: g_{2} \cdot \infty: g_{3} \cdot \infty\right]\right],
\end{gathered}
$$

also denoted by $\tau$.

Consider the map $k: B S L_{2}(\mathbb{C}) \rightarrow J\left(S^{2}\right)_{S L_{2}(\mathbb{C})}$ of (3) which is the map induced by the $S L_{2}(\mathbb{C})$-map $\ell: E S L_{2}(\mathbb{C}) \rightarrow J\left(S^{2}\right)$ of Corollary 3.5 on the sets of $S L_{2}(\mathbb{C})$ orbits.

Proposition 7.2. The homomorphism $k_{*}: H_{3}\left(B S L_{2}(\mathbb{C}), \mathbb{Z}\right) \rightarrow \mathcal{P}(\mathbb{C})$ induced by $k$ in homology is the homomorphism $\tau$ in (10).

Proof. From (2) we have that the map $k$ is given by (the geometric realisation of) the simplicial map

$$
\left(g_{0}, \ldots, g_{n}\right)_{S L_{2}(\mathbb{C})} \rightarrow\left(g_{0} \cdot \infty, \ldots, g_{n} \cdot \infty\right)_{S L_{2}(\mathbb{C})},
$$

which applied to 3 -simplices and composed with the isomorphism (8) is precisely the map $\tau(10)$.

The Bloch-Wigner exact sequence $[\mathbf{8}$, Appendix $\mathrm{A}]$ is given by

$$
0 \rightarrow \mathbb{Q} / \mathbb{Z} \rightarrow H_{3}^{E M}\left(S L_{2}(\mathbb{C}), \mathbb{Z}\right) \stackrel{\tau}{\rightarrow} \mathcal{P}(\mathbb{C}) \stackrel{\lambda}{\rightarrow} \wedge_{\mathbb{Z}}^{2}\left(\mathbb{C}^{\times}\right) \stackrel{\text { sym }}{\longrightarrow} K_{2}(\mathbb{C}) \rightarrow 0,
$$

where $\mathbb{Q} / \mathbb{Z}=H_{3}\left(\mu_{\mathbb{C}} ; \mathbb{Z}\right)$ with $\mu_{\mathbb{C}} \subset \mathbb{C}^{\times}$the group of roots of unity. The first map is induced by the inclusion of $\mu_{\mathbb{C}}$ into the diagonal of $S L_{2}(\mathbb{C})$. The homomorphism $\tau$ is the one given in (10). The homomorphism $\lambda$ was defined in (6) and for $u, v \in \mathbb{C}^{\times}$, $\operatorname{sym}(u \wedge v)=\{u, v\} \in K_{2}(\mathbb{C})$ denotes the $K_{2}$-symbol.

\section{The Bloch invariant lies in $B(\mathbb{C})$}

In the present section we prove that $\alpha(M)=\beta(M) \in B(\mathbb{C})$ by factoring the canonical homomorphism $\psi_{*}: H_{3}\left(M_{+} ; \mathbb{Z}\right) \rightarrow \mathcal{P}(\mathbb{C})$ through the homomorphism $\tau: H_{3}^{E M}\left(S L_{2}(\mathbb{C}), \mathbb{Z}\right) \rightarrow \mathcal{P}(\mathbb{C})$ of $(11)$. We do this constructing a map $R: M_{+} \rightarrow$ $B S L_{2}(\mathbb{C})^{+}$such that $\psi \simeq h \circ R$, where $h: B S L_{2}(\mathbb{C})^{+} \rightarrow J\left(S^{2}\right)_{S L_{2}(\mathbb{C})}$ is the map induced by the canonical map $k: B S L_{2}(\mathbb{C}) \rightarrow J\left(S^{2}\right)_{S L_{2}(\mathbb{C})}$ by the plus-construction. Therefore $\psi_{*}=\tau \circ R_{*}$. 


\subsection{Construction of the map $R$}

Let $G$ be a group and let $H$ be a perfect normal subgroup. Consider Quillen's plus construction relative to $H$. Let $\mathcal{F}$ be the homotopy fibre of

$$
\mathcal{F} \rightarrow B G \rightarrow B G^{+}
$$

then, $\mathcal{F}$ has the following properties (see $[\mathbf{1 6},(2.1)],[\mathbf{1 0}]$ )

1. $\mathcal{F}$ is connected and $\pi_{1}(\mathcal{F})$ is a universal central extension of $H$.

2. $\mathcal{F}$ is acyclic, i.e., $\tilde{H}_{\bullet}(\mathcal{F})=0$.

3. $\pi_{i}(\mathcal{F})=\pi_{i+1}\left(B G^{+}\right)$for $i \geqslant 2$.

4. The sequence (12) is both a fibration sequence and a cofibration sequence (see [10, Thm. (2.5)], [4, Thm. (7.7)]).

Let $M$ be a non-compact orientable complete hyperbolic 3-manifold of finite volume endowed with a spin structure. By Theorem $2.1 M$ is homotopically equivalent to $M_{0}$, and therefore, the representation $\rho: \Gamma \rightarrow S L_{2}(\mathbb{C})$ corresponds to a map

$$
f=B \rho: M_{0} \rightarrow B S L_{2}(\mathbb{C}) .
$$

Recall that $S L_{2}(\mathbb{C})$ is the group of elementary matrices $E_{2}(\mathbb{C})$ of $G L_{2}(\mathbb{C})$. Since $\mathbb{C}$ is a field, we have that $S L_{2}(\mathbb{C})=E_{2}(\mathbb{C})$ is a perfect group. Applying the plusconstruction to the space $B S L_{2}(\mathbb{C})$ we get the (co-)fibration (12) for $G=S L_{2}(\mathbb{C})$

$$
\mathcal{F} \stackrel{j}{\rightarrow} B S L_{2}(\mathbb{C}) \stackrel{p}{\rightarrow} B S L_{2}(\mathbb{C})^{+} .
$$

Let $\partial M_{0}$ be the boundary of $M_{0}$. Note that $M_{0} / \partial M_{0}$ is homeomorphic to the one-point compactification $M_{+}$of $M$. Consider the following diagram where $\iota: \partial M_{0} \rightarrow M_{0}$ is the inclusion and $q: M_{0} \rightarrow M_{+}$is given by collapsing $\partial M_{0}$ to a point as in (9).

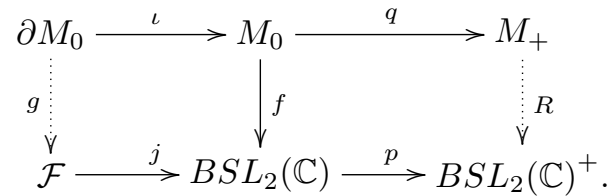

Our aim is to extend the map $p \circ f$ to a map $R: M_{+} \rightarrow B S L_{2}(\mathbb{C})^{+}$, this is possible if and only if the map $p \circ f \circ \iota$ is nullhomotopic and every extension $R$ corresponds to a nullhomotopy of $p \circ f \circ \iota$. The homotopy class of the extension $R$ only depends on $f$ and the nullhomotopy of $p \circ f \circ \iota[\mathbf{1 1}$, Lemma 11.2'].

Lemma 8.1. There is a one-to-one correspondence between nullhomotopies $H_{t}$ : po $f \circ \iota \sim *$ and pairs $\left(g, \tilde{H}_{t}\right)$ with $g: \partial M_{0} \rightarrow \mathcal{F}$ and $\tilde{H}_{t}$ a homotopy $\tilde{H}_{t}: f \circ \iota \sim j \circ g$.

Proof. Let $H_{t}$ be a nullhomotopy of $p \circ f \circ \iota$, since $p$ is a fibration we can lift $H_{t}$ to a homotopy $\tilde{H}_{t}$ such that $\tilde{H}_{0}=f \circ \iota$ and $p \circ \tilde{H}_{t}=H_{t}$ for all $t \in[0,1]$. Since $H_{1}$ is the constant map, the image of $\tilde{H}_{1}$ is contained in $\mathcal{F}$. Let $g=\tilde{H}_{1}$, hence $\left(g, \tilde{H}_{t}\right)$ is the pair corresponding to the nullhomotopy $H_{t}$.

Let $\left(g, \tilde{H}_{t}\right)$ with $g: \partial M_{0} \rightarrow \mathcal{F}$ and $\tilde{H}_{t}: f \circ \iota \sim j \circ g$. Since $p$ is a fibration and $j: \mathcal{F} \rightarrow B S L_{2}(\mathbb{C})$ is the inclusion of its fibre, $p \circ \tilde{H}_{t}$ gives the desired nullhomotopy of $p \circ f \circ \iota$. 
By Lemma 8.1 to define an extension $R$ is enough to give a map $g: \partial M_{0} \rightarrow \mathcal{F}$ such that the left square of (14) commutes up to homotopy and to choose a homotopy $\tilde{H}_{t}: f \circ \iota \sim j \circ g$.

The boundary $\partial M_{0}$ consists of a disjoint union of tori, we construct the map $g: \partial M_{0} \rightarrow \mathcal{F}$ on each torus component separately. The restriction of $f \circ \iota$ to a component $T_{i}^{2}$ of the boundary gives a map

$$
f_{i}=\left.f \circ \iota\right|_{T_{i}^{2}}: S^{1} \times S^{1} \rightarrow B S L_{2}(\mathbb{C}) .
$$

This map induces in homotopy the homomorphism

$$
\begin{aligned}
\mathbb{Z} \times \mathbb{Z} & \rightarrow S L_{2}(\mathbb{C}) \\
(m, n) & \mapsto A^{m} B^{n}
\end{aligned}
$$

for some commuting matrices $A, B \in S L_{2}(\mathbb{C})$, which are the generators of the corresponding peripheral torus subgroup $\Gamma_{i}$ of $\Gamma$. The matrices $A$ and $B$ corresponds to the homotopy classes of the loops given by the restrictions $\left.f_{i}\right|_{S^{1} \times\{*\}}: S^{1} \times\{*\} \rightarrow$ $B S L_{2}(\mathbb{C})$ and $\left.f_{i}\right|_{\{*\} \times S^{1}}:\{*\} \times S^{1} \rightarrow B S L_{2}(\mathbb{C})$.

We need to find maps $g_{i}: T_{i}^{2} \rightarrow \mathcal{F}$ which make the following diagram commute up to homotopy

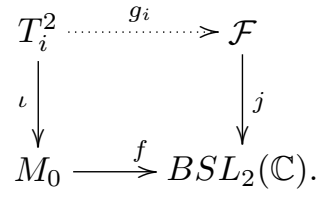

Since $S L_{2}(\mathbb{C})$ is a perfect group it has a universal central extension given by

$$
1 \rightarrow H_{2}\left(S L_{2}(\mathbb{C})\right) \rightarrow S t_{2}(\mathbb{C}) \stackrel{\varphi}{\rightarrow} S L_{2}(\mathbb{C}) \rightarrow 1,
$$

where $S t_{2}(\mathbb{C})$ is the Steinberg group. This universal central extension is induced by the homotopy exact sequence of the fibration $(13)$ and we have that $\pi_{1}(\mathcal{F})=S t_{2}(\mathbb{C})$ (property 1 of (12)).

Take loops $a, b: S^{1} \rightarrow \mathcal{F}$ such that $\varphi(a)=A$ and $\varphi(b)=B$. Then we can form the map

$$
a \vee b: S^{1} \vee S^{1} \rightarrow \mathcal{F}
$$

The obstruction to extend this map to a map $S^{1} \times S^{1} \rightarrow \mathcal{F}$ is given by the Whitehead product $[a, b]$ of $a$ and $b$ given by the commutator. Thus, we need to prove that $[a, b]$ vanish for any lifts $a, b \in S t(\mathbb{C})$ of $A$ and $B$ respectively, when $A$ and $B$ are the generators of a peripheral torus subgroup of $\Gamma$. For that, we need to recall a construction of elements in $K_{2}(\mathbb{C})=H_{2}\left(S L_{2}(\mathbb{C})\right)$ given in $[\mathbf{1 2}, \S 8]$.

Consider the universal central extension given in (16). Given two matrices $A, B \in$ $S L_{2}(\mathbb{C})$ which commute, choose representatives $a, b \in S t_{2}(\mathbb{C})$ such that $\varphi(a)=A$ and $\varphi(b)=B$. The commutator $[a, b]=a b a^{-1} b^{-1}$ is then an element of $K_{2}(\mathbb{C})$. We shall use Milnor's notation

$$
A \star B=a b a^{-1} b^{-1} \in K_{2}(\mathbb{C})
$$

for this commutator. The element $A \star B$ does not depend on the choice of representatives $a$ and $b$. 
As a direct consequence of identities satisfied by commutators the product $\star$ satisfies the following properties:

- Bimultiplicativity

$$
\left(A_{1} A_{2}\right) \star B=\left(A_{1} \star B\right)\left(A_{2} \star B\right)
$$

- Invariance under inner automorphisms of $S L_{2}(R)$

$$
\left(P A P^{-1}\right) \star\left(P B P^{-1}\right)=A \star B .
$$

By Remark 2.2 the matrices $A, B \in S L_{2}(\mathbb{C})$ represent parabolic isometries of $\mathcal{H}^{3}$ and they are conjugate to matrices of the form

$$
\bar{A}=\left(\begin{array}{cc}
\lambda & \delta \\
0 & \lambda
\end{array}\right), \quad \bar{B}=\left(\begin{array}{cc}
\mu & \varepsilon \\
0 & \mu
\end{array}\right)
$$

with $\lambda= \pm 1, \mu= \pm 1$, and $\delta \neq 0, \varepsilon \neq 0$. By property (19), to prove that $A \star B=1$ is enough to prove that $\bar{A} \star \bar{B}=1$ for all the possible values of $\lambda$ and $\mu$.

Proposition 8.2. Let $\bar{A}$ and $\bar{B}$ as above. Then $\bar{A} \star \bar{B}=1$.

Proof. By the bimultiplicativity (18) of the $\star$-product we have that

$$
\bar{A}^{2} \star \bar{B}^{2}=\left(\bar{A} \star \bar{B}^{2}\right)\left(\bar{A} \star \bar{B}^{2}\right)=(\bar{A} \star \bar{B})^{4} .
$$

By a result of Bass and Tate [3, Prop. 1.2] $K_{2}(\mathbb{C})$ is a uniquely divisible abelian group, i.e., a vector space over $\mathbb{Q}$. Therefore, if we prove that $(\bar{A} \star \bar{B})^{4}=1$, it will imply that $\bar{A} \star \bar{B}=1$. We have that

$$
\bar{A}^{2}=\left(\begin{array}{cc}
1 & 2 \lambda \delta \\
0 & 1
\end{array}\right)=e_{12}^{2 \lambda} \quad \bar{B}^{2}=\left(\begin{array}{cc}
1 & 2 \mu \varepsilon \\
0 & 1
\end{array}\right)=e_{12}^{2 \mu \varepsilon}
$$

where $e_{i j}^{a}$ denotes the elementary matrix with its $i j$ entry equal to $a$. Let $x_{i j}^{a}$ be the corresponding generator of the Steinberg group, thus,

$$
\bar{A}^{2} \star \bar{B}^{2}=x_{12}^{2 \lambda \delta} x_{12}^{2 \mu \varepsilon} x_{12}^{-2 \lambda \delta} x_{12}^{-2 \mu \varepsilon}=x_{12}^{2(\lambda \delta+\mu \varepsilon)} x_{12}^{-2(\lambda \delta+\mu \varepsilon)}=1 .
$$

We thank Michel Matthey for suggesting to use that $K_{2}(\mathbb{C})$ is uniquely divisible to simplify the computations. This proposition can also be proved without using this fact but one has to analyse the four cases separately (see [5]).

Therefore, by Proposition 8.2 the map (17) extends to a map $S^{1} \times S^{1} \rightarrow \mathcal{F}$ which makes the diagram (15) commute up to homotopy since $S^{1} \times S^{1}$ is a classifying space $B(\mathbb{Z} \times \mathbb{Z})$ and the maps $f_{i}$ and $j \circ g_{i}$ induce the same homomorphism on fundamental groups.

Taking all the maps $g_{i}: T_{i}^{2} \rightarrow \mathcal{F}$ together we get the desired map $g: \partial M_{0} \rightarrow \mathcal{F}$. Choose a homotopy $\tilde{H}_{t}: f \circ \iota \sim j \circ g$, since $\iota: \partial M_{0} \rightarrow M_{0}$ is a cofibration we can lift $\tilde{H}_{t}$ to a homotopy $\bar{H}_{t}$ such that $\bar{H}_{0}=f$ and $\bar{H}_{t} \circ \iota=\tilde{H}_{t}$ for all $t \in[0,1]$. Let 
$f_{1}=\bar{H}_{1}$, then we have the following strictly commutative diagram

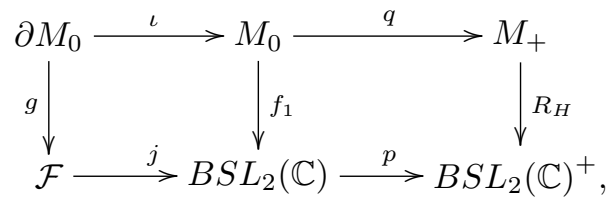

where

$$
R_{H}: M_{+} \rightarrow B S L_{2}(\mathbb{C})^{+}
$$

is the map defined by the nullhomotopy $H$ corresponding to $\left(g, \tilde{H}_{t}\right)$ by Lemma 8.1.

\section{2. $\quad$ Factoring $\psi$ through $R$}

Our next task is to prove that $\tau \circ R_{H_{*}}=\psi_{*}$. For this, we need the following lemmata.

Lemma 8.3. $\pi_{1}\left(J\left(S^{2}\right)_{S L_{2}(\mathbb{C})}\right)=0$.

Proof. It is a direct application of a result by M. A. Armstrong [1, Thm. 3], [2, Thm. 6.18] which says that $\pi_{1}\left(J\left(S^{2}\right)_{S L_{2}(\mathbb{C})}\right)=S L_{2}(\mathbb{C}) / F$ where $F$ is the normal subgroup of $S L_{2}(\mathbb{C})$ generated by those elements which leave fixed at least one point of $J\left(S^{2}\right)$. Obviously the elements of $S L_{2}(\mathbb{C})$ which are matrices of the form $\left(\begin{array}{ll}1 & a \\ 0 & 1\end{array}\right)$, with $a \in \mathbb{C}$ and of the form $\left(\begin{array}{ll}1 & 0 \\ b & 1\end{array}\right)$, with $b \in \mathbb{C}$ are in $F$, they fix $(\infty, \ldots, \infty)$ and $(0, \ldots, 0)$ respectively. But these are elementary matrices and the whole $S L_{2}(\mathbb{C})$ is generated by elementary matrices $\left(\right.$ since $S L_{2}(\mathbb{C})=E_{2}(\mathbb{C})$ ), therefore $F=S L_{2}(\mathbb{C})$.

Lemma 8.4. $\pi_{2}\left(J\left(S^{2}\right)_{S L_{2}(\mathbb{C})}\right)=0, \quad \pi_{3}\left(J\left(S^{2}\right)_{S L_{2}(\mathbb{C})}\right)=\mathcal{P}(\mathbb{C})$.

Proof. By the proof of $\left[\mathbf{1 7}\right.$, Lemma 2.2] we have that $H_{1}\left(J\left(S^{2}\right)_{S L_{2}(\mathbb{C})}\right)=0$, $H_{2}\left(J\left(S^{2}\right)_{S L_{2}(\mathbb{C})}\right)=0$ and $H_{3}\left(J\left(S^{2}\right)_{S L_{2}(\mathbb{C})} ; \mathbb{Z}\right) \cong \mathcal{P}(\mathbb{C})$. By Lemma $8.3 J\left(S^{2}\right)_{S L_{2}(\mathbb{C})}$ is simply connected and the lemma follows by Hurewicz Theorem.

Remember that a map between path connected spaces is acyclic if its homotopy fibre is an acyclic space. By the properties of (12) the homotopy fibration

$$
\mathcal{F} \stackrel{j}{\rightarrow} B S L_{2}(\mathbb{C}) \stackrel{p}{\rightarrow} B S L_{2}(\mathbb{C})^{+}
$$

is acyclic since $\mathcal{F}$ is acyclic. We have also that $j$ is a cofibration and $p$ is the cofibre of $j$.

By Corollary 3.5 there is a $S L_{2}(\mathbb{C})$-map $\ell: E S L_{2}(\mathbb{C}) \rightarrow J\left(S^{2}\right)$ unique up to $S L_{2}(\mathbb{C})$-homotopy which induces a map $k: B S L_{2}(\mathbb{C}) \rightarrow J\left(S^{2}\right)_{S L_{2}(\mathbb{C})}$ between the $S L_{2}(\mathbb{C})$-orbit spaces. Let $\pi_{1}(p)$ and $\pi_{1}(k)$ the homomorphisms on fundamental groups induced by $p$ and $k$, by Proposition 8.3 we have that $\operatorname{ker} \pi_{1}(p)=\operatorname{ker} \pi_{1}(k)=$ $S L_{2}(\mathbb{C})$ and by $[\mathbf{1 0}$, Prop. $(3.1)],[\mathbf{4},(5.2)]$ there exist a map $h: B S L_{2}(\mathbb{C})^{+} \rightarrow$ 
$J\left(S^{2}\right)_{S L_{2}(\mathbb{C})}$ unique up to homotopy which makes the following diagram commute

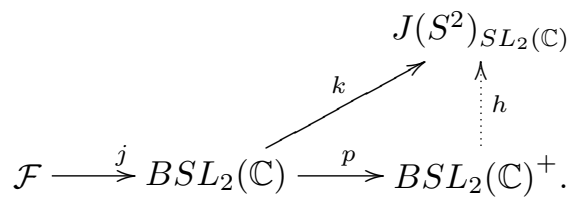

Remark 8.5. The map $h$ is also given by the functoriality of the plus-construction applied to the map $k$, that is $h=k^{+}$, since $J\left(S^{2}\right)_{S L_{2}(\mathbb{C})}^{+}=J\left(S^{2}\right)_{S L_{2}(\mathbb{C})}$.

Proposition 8.6. The following diagram commutes (up to homotopy)

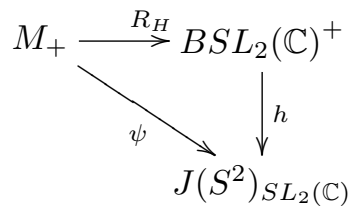

where $R_{H}$ is the map given in (21), $\psi$ is the canonical map (9) and $h$ is the map given in (22).

Proof. Firstly, we modify diagram (9) to make it compatible with diagram (20). Consider the homotopy $\bar{H}_{t}: f \sim f_{1}$ that we use in (20) and a homotopy $\bar{F}_{t}: k \sim h \circ p$. Define a homotopy $F_{t}: k \circ f \sim h \circ p \circ f_{1}$ by $\bar{F}_{t} \circ \bar{H}_{t}$. Since $q: M_{0} \rightarrow M_{+}$is a cofibration we can extend $F_{t}$ to a homotopy $\tilde{F}_{t}$ such that $\tilde{F}_{1}=\psi$ and $\tilde{F}_{t} \circ q=F_{t}$ for all $t \in[0,1]$. Let $\psi_{1}=\tilde{F}_{1}$, then we get the following strictly commutative diagram

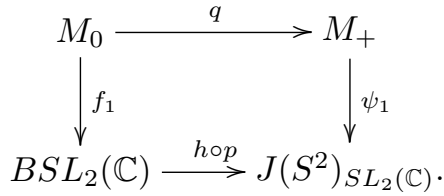

Putting together diagrams (20) and (23) we get

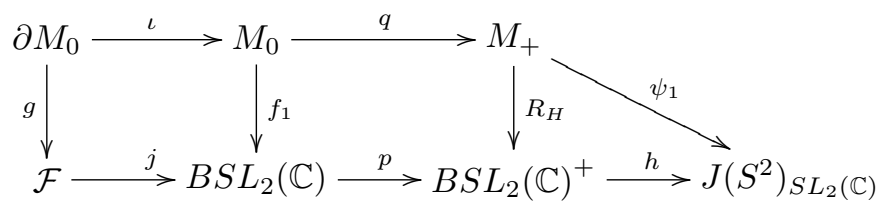

From diagram (20) we have that

$$
\begin{aligned}
f_{1} \circ \iota & =j \circ g, \\
R_{H} \circ q & =p \circ f_{1} .
\end{aligned}
$$

From diagram (23) we have

$$
\psi_{1} \circ q=h \circ p \circ f_{1} .
$$


We want to prove that the triangle in (24) commutes (up to homotopy), that is $\psi_{1} \simeq h \circ R_{H}$. Substituting (26) in (27) we obtain

$$
\psi_{1} \circ q=h \circ R_{H} \circ q .
$$

To simplify notation, put $v_{o}=\psi_{1}, v_{1}=h \circ R_{H}, G=S L_{2}(\mathbb{C})$ and $J=$ $J\left(S^{2}\right)_{S L_{2}(\mathbb{C})}$. Thus we have two maps $v_{i}: M_{+} \rightarrow J, i=0,1$, such that $v_{0} \circ q=v_{1} \circ q$. We want to prove that $v_{o} \simeq v_{1}$.

Let $F: B G \times I \rightarrow J$ given by $F(z, t)=h \circ p$ for all $t \in[0,1]$ then $H=F \circ\left(f_{1} \times\right.$ id) $: M_{0} \times I \rightarrow J$ is the constant homotopy between $v_{o} \circ q$ and $v_{1} \circ q$ since

$$
\begin{aligned}
& H(x, 0)=F(f(x), 0)=h \circ p \circ f_{1}(x)=\psi_{1} \circ q(x)=v_{0} \circ q(x) \\
& H(x, 1)=F(f(x), 1)=h \circ p \circ f_{1}(x)=h \circ R_{H} \circ q(x)=v_{1} \circ q(x) .
\end{aligned}
$$

Such homotopy is equivalent to a map $\tilde{H}: M_{0} \rightarrow J^{I}$, where $J^{I}$ denotes the continuous (free) maps from $I$ to $J$, given by $\tilde{H}(x)(t)=H(x, t)$. Hence we have the following commutative diagram

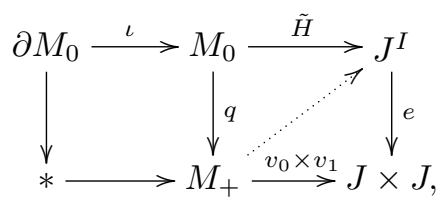

where $e$ is evaluation on $\{0,1\} \subset I$. To find a homotopy between $v_{0}$ and $v_{1}$ is equivalent to give a lifting of $v$ into $J^{I}$, this is possible if the pair of maps $(\tilde{H} \circ \iota, *)$ is nullhomotopic [11, Lemma 11.1'], where $*$ denotes the constant map to the basepoint. The map $e$ is a fibration with fibre $\Omega J$, the loop space of $J$, and since $e \circ \tilde{H} \circ \iota$ is the constant map, the map $\tilde{H} \circ \iota$ has its image in $\Omega J$. The condition that the pair of maps $(\tilde{H} \circ \iota, *)$ is nullhomotopic is equivalent to $\tilde{H} \circ \iota$ being nullhomotopic in $\Omega J$.

Putting $\tilde{F}: B G \rightarrow J^{I}$ as $\tilde{F}(z)(t)=F(z, t)$ we have the following commutative diagram

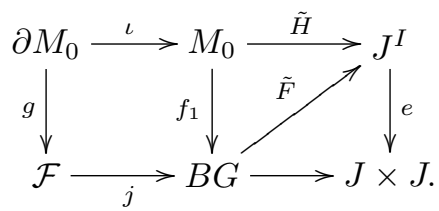

Since $e \circ \tilde{F} \circ j$ is also the constant map, the map $\tilde{F} \circ j$ also has its image in $\Omega J$, therefore we have the following commutative diagram

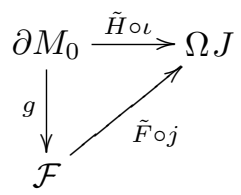

By lemma $8.4 \pi_{1}(\Omega J)=\pi_{2}(J)=0$. Hence the map induced on fundamental groups $\pi_{1}(\tilde{F} \circ j)$ is zero and since $\mathcal{F}$ is acyclic by [10, Cor. (3.2)] $\tilde{F} \circ j$ is nullhomotopic 
in $\Omega J$. Therefore $\tilde{H} \circ \iota$ is nullhomotopic in $\Omega J$ which implies that $v_{0}$ and $v_{1}$ are homotopic.

Now it is a corollary the fact that the invariant $\alpha(M)$ (and so the Bloch invariant) lies in the Bloch group, compare with $[\mathbf{1 3}, \S 1, \S 5]$.

Corollary 8.7. $\alpha(M) \in B(\mathbb{C})$.

Proof. The diagram of Proposition 8.6 induces the following commutative diagram in homology

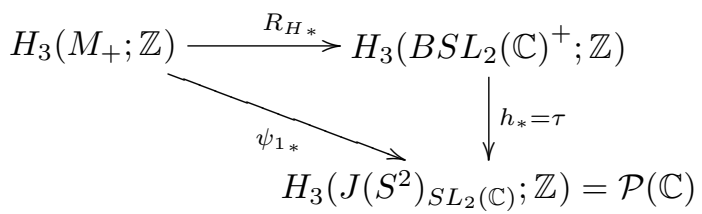

By Remark 8.5 we have that $h=k^{+}$, hence $h$ and $k$ induce the same homomorphism in homology. By Proposition 7.2 we have that $k_{*}=\tau$ where $\tau$ is the map (10). Hence $h_{*}=\tau$ and

$$
\alpha(M)=\psi_{1_{*}}\left(\left[M_{+}\right]\right)=\tau \circ R_{H *}([\hat{M}]) \in B(\mathbb{C}),
$$

since by the Bloch-Wigner exact sequence (11), the image of $\tau$ is the Bloch group $B(\mathbb{C})$.

Corollary 8.8. Let $M$ be a non-compact orientable complete hyperbolic 3-manifold of finite volume which has an ideal triangulation by ideal tetrahedra $\triangle_{1}, \ldots, \triangle_{n}$. Let $z_{i} \in \mathbb{C}$ be the parameter of $\triangle_{i}$ for each $i$. Then

$$
2 \sum_{i=1}^{n} z_{i} \wedge\left(1-z_{i}\right)=0 \in \wedge_{\mathbb{Z}}^{2}\left(\mathbb{C}^{\times}\right) .
$$

Proof. By Corollary 8.7 and the Bloch-Wigner exact sequence (11) $\beta(M)$ is in the kernel of the homomorphism $\lambda$ given in (6) and the left-hand-side of (29) is precisely $\lambda(\beta(M))$.

Remark 8.9. By a result of Sah [15, Prop. 2.9 (a), Thm. 4.1 (b)] we have that $K_{3}(\mathbb{C}) \cong H_{3}(S L(\mathbb{C}) ; \mathbb{Z}) \cong H_{3}\left(S L_{2}(\mathbb{C}) ; \mathbb{Z}\right) \oplus K_{3}^{M}(\mathbb{C})$ and $K_{3}^{\text {ind }}(\mathbb{C}) \cong H_{3}\left(S L_{2}(\mathbb{C}) ; \mathbb{Z}\right)$. Here $K_{3}^{M}(\mathbb{C})$ is Milnor's K-theory and $K_{3}^{\text {ind }}(\mathbb{C})$ is the idecomposable part of $K_{3}(\mathbb{C})$. We can construct an element in $K_{3}^{\text {ind }}(\mathbb{C})$ mapping the fundamental class $\left[M_{+}\right]$to $H_{3}\left(S L_{2}(\mathbb{C}) ; \mathbb{Z}\right)$ with the homomorphism $R_{H *}$ in $(28)$ and we have that such element is mapped to the Bloch invariant under $\tau$. Composing $R_{H}$ with the map $s: B S L_{2}(\mathbb{C})^{+} \rightarrow B S L(\mathbb{C})^{+}$induced by the inclusion, we get an element in $K_{3}(\mathbb{C})$ and it can be proved that such an element is also mapped to the Bloch invariant under a homomorphism $H_{3}(S L(\mathbb{C}) ; \mathbb{Z}) \rightarrow B(\mathbb{C})$ given by $\tau$.

The present paper is the result of an attempt to lift the Bloch invariant to $K_{3}(\mathbb{C})$ in this way. Unfortunately the map $R_{H}$ depends on a specific choice of nullhomotopy of $p \circ f \circ \iota$ in $(14)$, and there is a $\mathbb{Q} / \mathbb{Z}$ indeterminacy due to such choice. To get rid of 
the indeterminacy we should quotient $H_{3}\left(S L_{2}(\mathbb{C}) ; \mathbb{Z}\right)$ by $\mathbb{Q} / \mathbb{Z}$ but we get back the Bloch group $B(\mathbb{C})$ by the Bloch-Wigner exact sequence (11). Thus, to lift the Bloch invariant to $K_{3}(\mathbb{C})$ one should look for a canonical way to construct an element in $K_{3}(\mathbb{C})$ from $M$.

Acknowledgments: The first author would like to thank Michel Matthey for many fruitful discussions.

\section{References}

[1] M. A. Armstrong. On the fundamental group of an orbit space. Proc. Camb. Phil. Soc., 61:639-646, 1965.

[2] M. A. Armstrong. Basic Topology. Undergraduate Texts in Mathematics. Springer-Verlag, 1983.

[3] H. Bass and J. Tate. The Milnor ring of a global field. In Lecture Notes in Mathematics 342, 1973.

[4] A. J. Berrick. An Approach to Algebraic K-theory. Research Notes in Math. 56. Pitman, 1982.

[5] José Luis Cisneros-Molina. The regulator, the Bloch group, hyperbolic manifolds, and the $\eta$-invariant. $\mathrm{PhD}$ thesis, University of Warwick, Coventry, England, September 1998.

[6] Marc Culler and Peter B. Shalen. Varieties of group representations and splittings of 3-manifolds. Annals of Mathematics, 117(109-146), 1983.

[7] Warren Dicks, Peter H. Kropholer, Ian J. Leary, and Simon Thomas. Classifying spaces for proper actions of locally-finite groups. Preprint. arXiv:math.GR/0111283 v1, Nov 2001.

[8] Johan L. Dupont and Chih-Han Sah. Scissors congruences, II. Journal of Pure and Applied Algebra, 25:159-195, 1982.

[9] D. B. A. Epstein and R. Penner. Euclidean decomposition of non-compact hyperbolic manifolds. J. Diff. Geom., 27:67-80, 1988.

[10] Jean-Claude Hausmann and Dale Husemoller. Acyclic maps. L'Einsegnement Mathématique, 25:53-75, 1979.

[11] Peter Hilton. Homotopy Theory and Duality. Notes on mathematics and its applications. Gordon and Breach, New York, 1965.

[12] J[ohn] [W.] Milnor. Introducton to Algebraic K-Theory. Study 72. Princeton University Press, Princeton, New Jersey, 1971.

[13] Walter D. Neumann and Jun Yang. Bloch invariants of hyperbolic 3manifolds. Duke Math. J., 96(1):29-59, 1999.

[14] John G. Ratcliffe. Foundations of Hyperbolic Manifolds. Graduate Texts in Mathematics 149. Springer-Verlag, 1994.

[15] Chih-Han Sah. Homology of classical lie groups made discrete III. Journal of Pure and Applied Algebra, 56:269-312, 1989. 
[16] A. A Suslin. On the equivalence of K-theories. Communications in Algebra, 9(15):1559-1566, 1981.

[17] A. A. Suslin. $K_{3}$ of a field and the Bloch group. Proceedings of the Steklov Institute of Mathematics, 4:217-239, 1991.

[18] William P. Thurston. Three-Dimensional Geometry and Topology, volume 1. Princeton University Press, Princeton, New Jersey, 1997.

[19] Tammo tom Dieck. Transformation Groups and Representation Theory. Lecture Notes in Mathematics 766. Springer-Verlag, Berlin-Heidelberg, 1979.

[20] Tammo tom Dieck. Transformation Groups. Number 8 in Studies in Mathematics. Walter de Gruyter, Berlin, 1987.

This article may be accessed via WWW at http://www.rmi.acnet.ge/hha/ or by anonymous ftp at

ftp://ftp.rmi.acnet.ge/pub/hha/volumes/2003/n1a14/v5n1a14.(dvi,ps,pdf)

José Luis Cisneros-Molina jlcm@matcuer. unam.mx

Instituto de Matemáticas

UNAM, Unidad Cuernavaca

Av. Universidad s/n, Col. Lomas de Chamilpa

Cuernavaca, Morelos, 62210

MEXICO

John D. S. Jones jdsj@maths.warwick.ac.uk

Mathematics Institute

University of Warwick

Coventry CV4 7AL

England 